\title{
In reply to the letter to the Editor, "Morphological evaluation of median nerve: why ultrasound?"
}

\author{
Alhusain $\mathrm{Nagm}^{1,2}$ (i) $\cdot$ Ahmed A. Negm $^{3}$
}

Received: 6 June 2017 / Accepted: 29 June 2017 / Published online: 10 July 2017

(c) Springer-Verlag GmbH Germany 2017

\section{Dear Editor;}

We greatly appreciate the thoughtful comments by the authors of the letter to the Editor: morphological evaluation of median nerve: why ultrasound regarding our publication "Ultrasonography of a bifid median nerve causing carpal tunnel syndrome: MSUS or MRI, which is better?" [1]. The authors provide constructive comments for a patient presented with paresthesia in the median nerve territory and strength defect at the thenar muscles attributed to traumatic median nerve injury at the wrist, in whose ultrasound (US) examination led to a definitive diagnosis. We agree with the authors that US can provide an excellent morphological evaluation that supports the decision of the more appropriate treatment, as stated by our experienced neurosurgeon (AN) [1], to limit the surgical risks for better outcome. In addition, we also agree that US provides a high-resolution assessment of the peripheral nervous system with possible visualization of the very small nerves and their terminal branches [1,2].

In cases with traumatic nerve injury, discrimination between axonotmesis and neurotmesis is paramount [2].

This reply refers to the comment available at doi:10.1007/s00296-017-3766-6.

Alhusain Nagm

nagm@shinshu-u.ac.jp

1 Department of Neurosurgery, Shinshu University School of Medicine, 3-1-1 Asahi, Matsumoto 390-8621, Japan

2 Department of Neurosurgery, Al-Azhar University Faculty of Medicine-Nasr city, Cairo, Egypt

3 Department of Rheumatology, Physical Medicine and Rehabilitation, Faculty of Medicine, Al-Azhar University, Cairo, Egypt
Interestingly, based on studying the median nerve from the forearm down to its terminal branches at the level of the phalange-metacarpal joints, the authors were able to exclude neurotmesis and to prove normal ultrasonographic findings proximal and distal to the trauma site. Defining these features, by US, deliver additional data that might contribute in the treatment plan for such patients.

From the neurosurgical point of view, such novel diagnostic modality [1] can clarify hidden pathologies that offer meticulous preoperative assessment with subsequent accurate decision. We strongly recommend the routine application of ultrasonography in all entrapment neuropathies (including thoracic outlet syndrome) and traumatic nerve injuries.

This case is an instructive case for all neurosurgeons and rheumatologists.

Sincerely;

Authors

\section{References}

1. Negm AA, Nagm A, Altamimyh H, Ghanem M (2017) Ultrasonography of a bifid median nerve causing carpal tunnel syndrome: MSUS or MRI, which is better? Rheumatol Int. doi:10.1007/s00296-017-3670-0

2. Renna R, Coraci D, De Franco P, Erra C, Ceruso M, Padua L (2012) Ultrasound study is useful to discriminate between axonotmesis and neurotmesis also in very small nerves: a case of sensory digital ulnar branch study. Med Ultrason 14(4):352-354 